\title{
Anti Hu Syndrome With Atypical Electrodiagnostic Findings
}

\author{
Amtul Farheen ${ }^{\mathrm{a}}$, Aiesha Ahmed ${ }^{\mathrm{b}, \mathrm{c}}$
}

\begin{abstract}
Anti-Hu syndrome is a paraneoplastic process associated with lung malignancy. Patients usually present with a sensory neuropathy, cerebellar syndrome or limbic encephalitis. Although motor involvement is described it is relatively rare and forms part of a more complex presentation with multiple systems affected.
\end{abstract}

Keywords: Dysesthesia; Weakness; Paraneoplastic process

\section{Introduction}

Anti Hu syndrome is usually associated with small cell lung cancer. Patients usually present with a sensory neuropathy, cerebellar syndrome or limbic encephalitis. In anti-Hu syndrome there is thought to be disseminated inflammation of brain, brainstem, spinal cord (lower motor neuron) and dorsal root ganglion. Our patient is the only reported case of anti -Hu syndrome where the electrodiagnostic data is very different from the clinical presentation. In our patient the initial complains were of dysesthesia and subsequently weakness but electrodiagnostic data only showed lower motor neuron involvement.

\section{Case Report}

A 55 year-old right-handed woman with history of chronic

Manuscript accepted for publication June 19, 2012

${ }^{a}$ NJ Neuroscience Institute, Seton Hall University, Edison, NJ 08820, USA

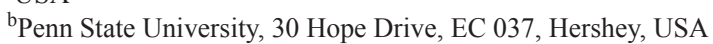

corresponding author: Aiesha Ahmed. Email: aahmed1@hmc.psu.edu

doi:10.4021/jnr111w smoking presented with almost 7 weeks history of burning pain and weakness that started a week after her gallbladder surgery. Initially, the pain was in the left hip and later it radiated to her leg and ankle, and the pain was described as sharp and throbbing. Later she developed dizziness and intermittent double vision. The dizziness went away however the double vision persisted. Diplopia was described as seeing two images either side by side or sometimes one on top of the other. The double vision was present in all directions. Subsequently, she noticed that she had difficulty walking. She started using a walker. Within a week, she noticed difficulty with gripping objects followed by difficulty lifting her arms up. Weakness had involved both sides but was more noticeable to her on the right. She also developed neck weakness which was rapidly progressive to the point that she was unable to lift her head off the pillow. During the course of all the above, she noticed sharp pain in different regions of her body. She also developed bilateral hand tremors. She denies muscle twitching, shrinkage of muscle, and muscle spasms. She denied any numbness or tingling. She denied any speech impairment. She had no bowel or bladder incontinence. She reported no weight loss, joint swelling, fever or chills.

Her past medical history was significant for hypertension, and coronary artery disease. Her family history was unremarkable. She smoked one pack a day for 15 years and denies drinking or illicit drug use.

On exam she was alert and oriented to time, place and person. Her pupils were equal and reactive with normal accommodation. Extraocular movements were normal bilaterally. No ptosis was seen at rest or on sustained upgaze for 30 seconds. She had normal eye closure strength. Facial sensation and strength were intact. Normal palatal elevation and tongue protrusion were noted. The motor examination revealed neck flexion strength graded as 0 and extension as $3+$. In the upper extremities strength is grades as follows (right/ left): Spinati $2 / 2$, deltoids $3 / 3$, biceps $3 / 3+$, triceps $3 / 5-$, wrist extensors $0 / 3+$, and interossei $0 / 3+$. In the lower extremities, strength was graded as follows (right/left): Hip flexors $3 / 3+$, knee flexors $4 / 4+$, Knee extensors $4-/ 5$, thigh adductors and abductors 5-/5-, foot dorsiflexors $0 / 4+$, foot plantar flexors $4 / 4$, foot eversion $0 / 0$ and inversion $4+/ 4+$. Her reflexes were 3 in the upper extremities and $3+$ at knees with positive 
cross adductors and 2 at the ankles bilaterally. Babinski sign was mute and Hoffman sign was absent bilaterally. Sensory examination revealed decreased pinprick in stocking distribution (mid-calf) and hyperesthesia in glove distribution (below the elbow level) bilaterally. Vibration and position sense was intact symmetrically. She has bilateral hand tremors. She was unable to get up from supine position without support.

Blood work including $\mathrm{CBC}$, chemistry, CK, Sedimentation rate, ANA complete panel, ANCA, B12 level, Serum and urine protein electrophoresis, ACE level, Copper level, Lyme titers, Thyroid panel, Acetylcholine receptor antibodies were unremarkable or normal. She was tested negative for HIV. Cerebrospinal fluid (CSF) analysis showed high proteins at 142, RBC 0 and WBC count of 23 (100\% mononuclear cells). CSF IgG index was 1.08. CSF RPR, HSV, CMV, Lyme were negative.

MRI of the brain and neuraxis were normal. NCS/ EMG were done and the findings were consistent with a diffuse process involving motor neurons and their axons (Table 1, 2).

As the patient was deteriorating very quickly, paraneoplastic panel was sent. Anti MATA was negative but Anti Hu was positive in titre of 1:160. CT chest was suspicious for right upper lobe mass and extensive right lower lobe infiltrate. Bronchoscopic biopsy of the lung mass was suspicious for small cell cancer and definitive diagnosis of small cell lung cancer was made after open lung biopsy. Two weeks after the onset of her presentation, the patient went into respiratory distress and needed to be intubated. She eventually had a tracheostomy and a gastrostomy tube placement. She was treated with plasmapharesis which did not show any improvement. Before cancer treatment could be initiated she expired.

\section{Discussion}

Anti $\mathrm{Hu}$ syndrome is usually associated with small cell lung cancer. $\mathrm{Hu}$ is RNA binding protein present in the nuclei of neurons, which is also expressed by tumor cells. An assumed pathophysiology is cross reactivity of host's immune response against the tumor with the host nervous system. An indicator that immune response controls tumor growth is that in many patients the paraneoplastic syndrome presents before the diagnosis is made. Patients usually present with a sensory neuropathy, cerebellar syndrome or limbic encephalitis. Although motor involvement is described it is relatively rare and forms part of a more complex presentation with multiple systems affected [1]. Loss of dorsal root ganglion cells leading to sensory neuronopathy is well described in the literature. Also, in anti-Hu syndrome there is thought to be disseminated inflammation of brain, brainstem, spinal cord and dorsal root ganglion [2]. Motor neuron degeneration in

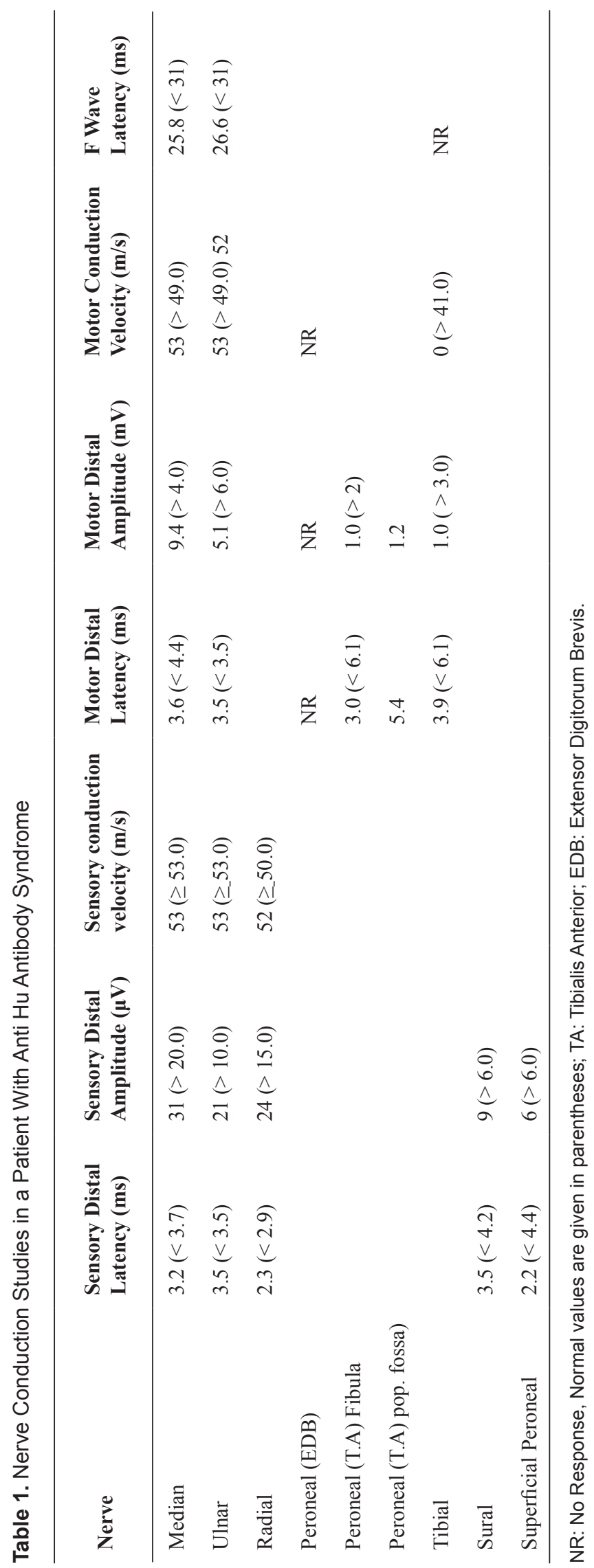


Table 2. Needle EMG Examination in a Patient With Anti-Hu Antibody Syndrome

\begin{tabular}{|c|c|c|c|c|c|c|c|c|c|}
\hline Muscle & Inser & Fibs & +Wave & Fasc & Dur & Amp & Poly & Recr & Effort \\
\hline First dorsal interosseus. L & $\mathrm{N}$ & None & None & None & $\mathrm{N}$ & $\mathrm{N}$ & None & $\mathrm{N}$ & Max \\
\hline Extensor digitorum communis. L & $\uparrow$ & $1+$ & $1+$ & None & $\mathrm{N}$ & $\mathrm{N}$ & None & $\mathrm{N}$ & Max \\
\hline Biceps brachii. L & $\uparrow$ & $2+$ & $2+$ & None & S.I & S.I & None & $\downarrow \downarrow$ & Max \\
\hline Triceps. L & $\uparrow$ & $1+$ & $1+$ & None & S.I & S.I & None & $\downarrow$ & Max \\
\hline Deltoid. L & $\uparrow$ & $3+$ & $3+$ & None & G.I & G.I & None & $\downarrow \downarrow$ & Max \\
\hline Genioglossus. L & $\mathrm{N}$ & None & None & None & $\mathrm{N}$ & $\mathrm{N}$ & None & $\mathrm{N}$ & Max \\
\hline Masseter. L & $\mathrm{N}$ & None & None & None & $\mathrm{N}$ & $\mathrm{N}$ & None & $\mathrm{N}$ & Max \\
\hline Tibialis Ant. L. & $\uparrow$ & $1+$ & $1+$ & None & S.I & S.I & None & $\downarrow$ & Max \\
\hline Gastrocnemius. L. & $\uparrow$ & $1+$ & $1+$ & None & $\mathrm{N}$ & $\mathrm{N}$ & None & $\downarrow$ & Max \\
\hline Vastus Medialis. L & $\uparrow$ & $2+$ & $2+$ & None & S.I & S.I & None & $\downarrow \downarrow$ & Max \\
\hline Iliopsoas. L & $\mathrm{N}$ & None & None & None & S.I & S.I & None & $\downarrow$ & Max \\
\hline Gluteus medius. L & $\uparrow$ & $1+$ & $1+$ & None & S.I & S.I & None & $\downarrow$ & Max \\
\hline Cervical paraspinals. L & $\uparrow$ & $2+$ & $2+$ & None & & & & & \\
\hline Cervical Paraspinals. $\mathrm{R}$ & $\uparrow$ & $2+$ & $2+$ & None & & & & & \\
\hline T6 paraspinal. $\mathrm{R}$ & $\uparrow$ & $2+$ & $2+$ & None & & & & & \\
\hline T6 paraspinal. $\mathrm{R}$ & $\uparrow$ & $2+$ & $2+$ & None & & & & & \\
\hline
\end{tabular}

Inser: Insertional; Fasc: Fasiculation; Dur: Duration; Amp: Amplitude; Recr: Recruitment; L.: Left; R.: Right; S.I: Slightly increased; G.I: greatly increased.

the spinal cord has been reported and is thought to cause the motor deficit seen clinically [2-4]. The electrophysiological data that is reported shows that axonal neuropathy is seen most frequently. Neuropathy is noted to be sensory $70 \%$ of the times followed by sensorimotor $(25 \%)$ and then pure motor (5\%) [5]. Camdessanche et al report data on a group of patient with anti-Hu syndrome who clinically presented with subacute sensory neuropathy and electrodiagnostically had sensory as well as motor nerve involvement [5]. In contrast, our patient presented with what seemed like subacute sensorimotor neuropathy but electrodiagnostically showed involvement of only motor neuron, their axons or both. To our knowledge, our patient is the only reported case of anti - $\mathrm{Hu}$ syndrome where the electrodiagnostic data is very different from the clinical presentation. Our patient had pain, paresthesia and weakness clinically and had electrodiagnostic data suggestive of a motor neuron disease process. Forsyth et al have reported that upto $14 \%$ of the patients with anti-Hu associated paraneoplastic syndrome have motor weakness as presenting symptoms and that eventually $20 \%$ of them will have lower motor neuron involvement [6]. However, in our patient the initial complains were of dysesthesia and she later developed weakness but electrodiagnostically we only noted lower motor neuron involvement.

In the appropriate clinical setting, anti-Hu syndrome should be considered when evaluating patients with electrodiagnostic findings of motor neuron disease with clinical presentation suggestive of sensorimotor neuropathy. As these patients almost always have symptoms of involvement of other areas of the nervous system and motor neuron disease is a fragment so they should be tested for anti-Hu antibodies. 


\section{Competing Interest}

No conflicts of interest exist for the author.

\section{References}

1. Schulz U, Randalls B, Counsell C. Anti-Hu syndrome: a rare presentation and a very difficult decision. Pract Neurol. 2007;7(5):336-341.

2. Eggers C, Hagel C, Pfeiffer G. Anti-Hu-associated paraneoplastic sensory neuropathy with peripheral nerve demyelination and microvasculitis. J Neurol Sci. 1998;155(2):178-181.

3. Henson RA, Urich H. Encephalomyelitis with car- cinoma. In: Henson RA, Urich H. Cancer and the nervous system. Oxford: Blackwell Scientific: 1982. 314-45.

4. Verma A, Berger JR, Snodgrass S, Petito C. Motor neuron disease: a paraneoplastic process associated with anti-hu antibody and small-cell lung carcinoma. Ann Neurol. 1996;40(1):112-116.

5. Camdessanche JP, Antoine JC, Honnorat J, Vial C, Petiot P, Convers P, Michel D. Paraneoplastic peripheral neuropathy associated with anti-Hu antibodies. A clinical and electrophysiological study of 20 patients. Brain. 2002;125(Pt 1):166-175.

6. Forsyth PA, Dalmau J, Graus F, Cwik V, Rosenblum MK, Posner JB. Motor neuron syndromes in cancer patients. Ann Neurol. 1997;41(6):722-730. 